\title{
Kollektivität im Gesundheitswesen: Ethische Theorien und Praxisfelder von Gruppen als Akteuren
}

\section{Anlässlich der Jahrestagung der Akademie für Ethik in der Medizin 2019 in Göttingen}

\author{
Silke Schicktanz
}

Online publiziert: 17. April 2019

(C) Springer-Verlag GmbH Deutschland, ein Teil von Springer Nature 2019

\begin{abstract}
„Den Wald vor lauter Bäumen nicht sehen“, ist eine altbekannte Redeweise und meint letztlich, dass man etwas Offensichtliches, nämlich das Ganze, übersieht, weil man den Blick nur auf Einzelheiten richtet. Überträgt man diese Erkenntnis auf die aktuelle Medizin- und Pflegeethik, so fällt doch auf, dass üblicherweise das Individuum weithin im Mittelpunkt unserer Diskussionen und Überlegungen steht. Sowohl in theoriegeladenen Debatten als auch in der angewandten klinischen Problemreflexion steht das Individuum in der Rolle als Patient*in, Arzt bzw. Ärztin, Pflegende*r, Forscher*in oder Bürger im Fokus der modernen Ethik und insbesondere dem modernen, liberalen Recht (Beier et al. 2016).

Der Zusammenschluss von Individuen zu einer eigenständig agierenden Gruppe wird allerdings bei Themen der allgemeinen Gesundheitsvorsorge oder der Rolle von Ethik-Gremien offensichtlich. Je nach thematischer Ausrichtung wird deutlich, dass der Fokus auf den Einzelnen nicht ausreicht, wenn es um Kollektiveinheiten wie die Öffentlichkeit, die Gesellschaft, die Klinik, das Unternehmen, die Krankenversicherung, die Kirche und nicht zuletzt den Staat als moralische Akteure oder Adressaten in gesundheitsethischen Diskussionen geht. Aber wie differenziert, reflektiert und begründet sind eigentlich unsere ethischen Prinzipien, Ansätze und Überlegungen im Hinblick auf Kollektive und Kollektivität?

Politisch-ethische und sozialethische Ansätze haben hierzu bereits Einiges entwickelt. Auch Disziplinen wie die Sozialphilosophie und Soziologie nehmen selbstverständlich Kollektivakteure in den Blick. Kollektive bzw. Kollektivakteure sind Gruppen von Personen, die sich durch gemeinschaftliches Handeln und gruppenorientierte Interessen auszeichnen. Ethische Überlegungen gehen dabei davon aus, dass
\end{abstract}

Prof. Dr. S. Schicktanz $(\bowtie)$

Institut für Ethik und Geschichte der Medizin, Universitätsmedizin Göttingen,

Humboldtallee 36, 37073 Göttingen, Deutschland

E-Mail: silke.schicktanz@medizin.uni-goettingen.de 
ein ethisch relevantes Kollektiv einen gemeinsamen „spirit“, einen Kollektivgeist bzw. kollektive Intentionen im Sinne einer geteilten Zielsetzung ausbildet (Schmid und Schweikard 2009). Dies kann über gemeinsame Verständigung, Austausch von Argumenten und Bildung gemeinschaftlicher Interessen geschehen. Hierbei spielen auch Identifikation, Vertrauen und geteilte Erfahrung eine große Rolle. Aber welche konkreten Verbindlichkeiten daraus entstehen und welche Rolle dabei Freiwilligkeit (durch Berufswahl, politisches Engagement) oder eher Zwangszugehörigkeit zu einer Gruppe (Kategorisierung als „Patient“ oder „Mensch mit Behinderung“) ausmachen, bleiben wichtige Fragen, die für die ethische Reflexion auf die Gesundheitsversorgung enorm relevant sind.

Offensichtlich gibt es auch ethische Konflikte zwischen den Interessen Einzelner und den Interessen einer Gruppe. Dies wird besonders deutlich am aktuell diskutierten Thema der Impfpflicht. Hier konfligieren nicht die Interessen von Individuen mit denen anderer Individuen, sondern individuelle Interessen mit dem kollektiven Interesse an der „Herdenimmunität“. Ethische Fragen nach Solidarität und sozialer Verantwortung für die Allgemeinheit müssen hier gegenüber dem Individualrecht auf körperliche Selbstbestimmung abgewogen und bewertet werden.

Im klinischen Alltag treffen oft Gruppen in ethischen Konfliktsituationen aufeinander, wie zum Beispiel in der Psychiatrie oder am Lebensende auf der Intensivstation, wo ein interprofessionelles Team mit dem Kranken und mit seiner Familie interagiert. Alle sehen ein, dass sie gemeinsam eine Entscheidung treffen müssen, mit der auch alle einverstanden sein sollten. Ein anderes Beispiel ist die Reproduktionsmedizin: Hier geht es fast immer um mehr als nur eine Person, nämlich um ein Paar oder eine Familie und das gemeinsame Interesse an einer Zeugung oder Fortführung des familiären Verbunds.

Selbst in Pharma-Skandalen - angefangen mit dem historischen Fall um Contergan bis zur aktuellen Diskussion um Lunapharm - wird deutlich, dass der moralische Fingerzeig auf Einzelne nicht immer ausreichend ist. So erhebt sich aufs Neue die Frage, ob und wie der dahinterstehende Konzern oder auch Behörden, und nicht nur einzelne Mitarbeiter, konkrete Verantwortung übernehmen müssen.

Die Rolle des Patienten als moralischer Akteur oder moralischer Adressat sowie das Konzept der Patientenautonomie gilt es ebenfalls um die kollektive Dimension zu erweitern (Schicktanz und Jordan 2013). Nach welchen Kriterien sollten Gremien Vertreter von Patientengruppen auswählen - reicht hier ein Zufallsprinzip oder sollte eine offizielle Repräsentanz vorliegen? Welche ethischen Anforderungen müssen Patienten oder Betroffene erfüllen, die sich in Vereinen oder Organisationen freiwillig zusammenschließen und sich durch Petitionen in der Gesundheitspolitik engagieren, um für ein Patientenkollektiv zu sprechen - und nicht nur für sich selbst?

In unserem hochkomplexen Gesundheitswesen agieren, gerade auf gesundheitsund biopolitischer Ebene, vorrangig gesellschaftlich organisierte Gruppen: Ärzteschaft, Industrieverbände, Patientenverbände, zivilgesellschaftliche Gruppen, Kirchen, Gewerkschaften, Interessensverbände von Pflegenden oder anderen Berufsgruppen (neben dem Staat als übergeordnetem Akteur). Insbesondere Expertengruppen, wie zum Beispiel Ethikgremien, stellen mit ihrem kollektiven Konsens oder ihren gemeinschaftlichen Kompromissen häufig wichtige Weichen für politische Entscheidungsprozesse. Meinen also Begriffe wie „der Staat“, „die Familie“, „der 
Konzern“, „die Patienten“ nur, dass sich hier eine bestimmte Anzahl Einzelner zusammenfindet, die sich quasi aufaddieren und die gleichen Interessen, Rechte oder Pflichten (zufällig?) teilen? Und könnten diese Gruppierungen samt ihren normativen Anliegen letztlich wieder in die von Individuen aufgeteilt werden?

Oder müssen wir nicht vielmehr diesen Begrifflichkeiten durch ihre übergeordnete Struktur eine eigenständige Bedeutung in der Ethik geben? Natürlich wirft das zugleich wichtige Fragen für die Ethiktheorie auf: Ab wann ist eine Zusammenkunft von Einzelnen eine „Gruppe“ bzw. wodurch zeichnet sich Austausch, Entscheiden und Verantworten in Gemeinschaften aus? Welche ethischen Prinzipien und Theorien brauchen wir, um diese sozialen Prozesse zu analysieren und zu reflektieren?

Auf der Jahrestagung der Akademie für Ethik in der Medizin vom 26.-28. September 2019 in Göttingen wollen wir uns diesen Fragen in ihrer ganzen Spannbreite widmen. Diese reicht von konkreten Diskussionen um die Impfpflicht oder um die Solidarität als kollektivem Wert für die Forschungsteilnahme bis hin zu theoretischer Differenzierung aus Ethik, Sozialwissenschaften, Theologie, Pflegewissenschaften, Gesundheitsversorgung und Recht, was kollektive Akteure ausmacht, wie kollektive moralische Ansprüche zustande kommen und gerechtfertigt werden können und inwiefern dies für die politische und soziale Dimension der Medizinethik relevant wird. Schließlich wird auch die aktuelle Debatte um Bürger-, Patienten- und Stakeholder-Beteiligung vertieft: Für wen und warum sind Gruppenvertretungen wichtig? Welche ethischen und praktischen Limitierungen sind bei solchen Beteiligungsprozessen zu bedenken? Welchen Beitrag kann die ethische Expertise leisten, um diese Beteiligungsprozesse $\mathrm{zu}$ gestalten?

Das Ganze ist also mehr als seine Einzelteile, und dennoch darf das Detail nicht fehlen. Forschende als Gruppe im Blick zu behalten und dabei die Einzelbedürfnisse nicht aus dem Auge zu verlieren - so ist unser Motto für die Durchführung der diesjährigen Jahrestagung. Das lokale Tagungsteam wird sich bemühen, dass dies auch für das soziale Miteinander im Rahmenprogramm der Tagung stimmt.

Alles in allem gibt es also viele Gründe, im September nach Göttingen zu kommen. Das Team der AG Ethik und Kultur der Biomedizin am Institut für Ethik und Geschichte der Medizin in Göttingen freut sich schon auf Sie! Unser Dank gilt schon im Voraus auch unseren Kooperationspartnern und Unterstützern, Alfred Simon, Claudia Wiesemann und dem Zentrum für Medizinrecht an der Universität Göttingen.

Interessenkonflikt S. Schicktanz gibt an, dass kein Interessenkonflikt besteht.

\section{Literatur}

Beier K, Jordan I, Wiesemann C, Schicktanz S (2016) Understanding collective agency in bioethics. Med Health Care Philos 19(3):411-422. https://doi.org/10.1007/s11019-016-9695-4

Schicktanz S, Jordan I (2013) Kollektive Patientenautonomie: Theorie und Praxis eines neuen bioethischen Konzepts. In: Simon A, Wiesemann C (Hrsg) Patientenautonomie. Mentis, Paderborn, S 287-302

Schmid HB, Schweikard DP (2009) Einleitung: Kollektive Intentionalität. Begriff, Geschichte, Probleme. In: Schmid HB, Schweikard DP (Hrsg) Kollektive Intentionalität. Eine Debatte über die Grundlagen des Sozialen. Suhrkamp, Frankfurt am Main, S 11-65 